\title{
Towards Scarless Surgery: An Endoscopic-Ultrasound Navigation System for Transgastric Access Procedures
}

\author{
Raúl San José Estépar ${ }^{1}$, Nicholas Stylopoulos ${ }^{2}$, Randy E. Ellis ${ }^{1}$, Eigil Samset ${ }^{1}$, \\ Carl-Fredrik Westin ${ }^{1}$, Christopher Thompson ${ }^{1}$, and Kirby Vosburgh ${ }^{1,2,3}$ \\ ${ }^{1}$ Brigham and Women's Hospital \\ ${ }^{2}$ Massachusetts General Hospital \\ ${ }^{3}$ Center of Integration of Medicine and Innovative Technology (CIMIT), Boston, MA \\ \{rjosest, ellis, samset, westin\}@bwh.harvard.edu, \\ \{nstylopoulos, ccthompson, kvosburgh\} apartners.org
}

\begin{abstract}
Scarless surgery is a new and very promising technique that can mark a new era in surgical procedures. We have created and validated a navigation system for endoscopic and transgastric access interventions in in vivo pilot studies. The system provides augmented visual feedback and additional contextual information by establishing a correspondence between the real time endoscopic ultrasound image and a preoperative $\mathrm{CT}$ volume using rigid registration. The system enhances the operator's ability to interpret the ultrasound image reducing the mental burden used in probe placement. Our analysis shows that rigid registration is accurate enough to help physicians in endoscopic abdominal surgery where, by using preoperative data for context and real-time imaging for targeting, distortions that limit the use of only preoperative data can be overcome.
\end{abstract}

\section{Introduction}

For centuries, the peritoneal cavity has been approached through large incisions of the anterior abdominal wall. In the past two decades, the laparoscopic approach has gained wide acceptance because it offers a safe and less invasive alternative: pain and the complications associated to large abdominal incisions are minimized. To further reduce the invasiveness of peritoneal access, the next logical step is to eliminate the incision through the abdominal wall altogether. Rather, natural orifices may provide the entry points for surgical interventions. Recently, several research groups have been able to access the peritoneal cavity through peroral- transgastric (i.e. through a small incision in the gastric wall) and also peranal-transcolonic approaches to perform organ resections in an animal model [1]. This new approach has the potential to replace or augment laparoscopic techniques currently used to treat many diseases. It may be especially beneficial to obese patients or those who have undergone multiple procedures and thus are at risk for adhesions.

Minimally invasive peroral-transgastric and peranal-transcolonic surgery is in its infancy. Before widespread use of these techniques is possible, several technical barriers must be overcome. These include providing the physician with adequate visual feedback, clear indicators of instrument location and orientation and support in the recognition of anatomic structures. The appearance of the abdominal structures 


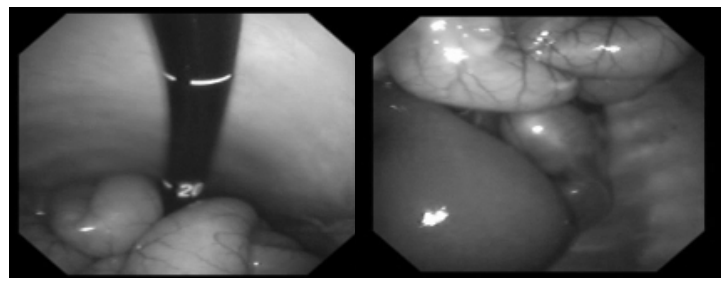

Fig. 1. Endoscopic view of a transgastric procedure

through the transgastric approach is totally different than in an open or laparoscopic approach (see Fig. 1). The unique angle of view, limited light, and the need to insert all instruments through a narrow channel are critical technical challenges. The introduction of the instruments in the peritoneal cavity (e.g. through a gastrotomy) should be performed in a way that precludes damage to surrounding organs and vasculature.

One relatively simple approach to augment the endoscopic/laparoscopic view is to use ultrasound (US). Laparoscopic US (LUS) and endoscopic US (EUS) are often used to guide biopsies and interventional procedures. However, the vast majority of physicians are not comfortable with performing invasive procedures under ultrasound guidance because of the inherent problems of image interpretation.

The navigation of a flexible endoscopic device inside the abdomen brings similar challenges as in traditional laparoscopy, but new complexities are added:

- The flexibility of the endoscope tip makes the understanding of its distal orientation difficult. Unlike laparoscopic procedures, there is no direct observation of the endoscope tip. Because of the lack of a global reference of the tip with respect to the patient body, successful navigation inside the stomach and in the abdomen cavity generally requires the expertise of a highly trained gastroenterologist (up to two years sub-specialization).

- A majority of the structures that are accessible through a transgastric access lie in a retrograde position with respect to the incision in the stomach wall. Access to such locations requires detailed knowledge of the place of the tip with respect to adjacent structures, particularly vessel.

- Understanding the position and orientation of the ultrasound B-scan plane is a ubiquitous problem even for experienced sonographers.

- An inherent problem to any ultrasound based system is the difficulty in interpreting the images because of low contrast, reduced field of view and acoustic windows constrains, despite the close proximity of the US probe to the target organs.

Several groups have attempted to address the orientation and interpretation problem by using preoperative data jointly with the intraoperative US data [2-6]. Lindseth et al. have shown that the fusion of intraoperative US images and preoperative MRI enhance the perception by extending the overview of the operating field. Ellsmere et al. showed that a 3D display with the main vascular structures and the probe positioning improved the spatial orientation of the operator, thus reducing the time to locate the organ of interest and increasing the operator's certainty. 
We present here a system that addresses those challenges and makes intra-cavitary interventional techniques easier to master and use in practice, and thus more likely to be widely adopted. Our system relies on providing context information about the interpretation of the US image based on preoperative data (CT or MRI). The system is based on tracking the endoscope tip and US plane with an electromagnetic tracker and established the correspondence of the real time positioning of the instrument tip with respect to preoperative data. The preoperative data is also used to generate $3 \mathrm{D}$ models of reference anatomical structure. Those structures are displayed with respect to the position of the probe in real time. An enhance interpretation of the US image is achieved by oblique reformatting the preoperative dataset according to the US plane.

\section{Navigation System}

The system consists of three major hardware components:

1. A conventional ultrasound machine equipped with a laparoscopic or endoscopic probe.

2. Tracking device comprising a transmitter and receiver sensors.

3. A host computer with a display for the use of the physician.

We use four coordinate systems:

- Instrument coordinate system $(D)$ : local to the device that is being tracked, namely, e.g. the tip of an endoscopic probe or a surgical pointing device.

- $\quad$ Receiver coordinate system $(R)$ : local to the sensor mounted on the tracked instrument.

- $\quad$ Transmitter coordinate system $(T)$ : The coordinate frame of the transmitter of the tracking device, taken as the global coordinate frame.

- Patient coordinate system $(P)$ : local to the patient in the CT scanner; intraoperatively this is also the coordinate frame of the display.

These coordinate frames are related by affine transformations that are provided either by the tracking system or by computations. Notationally, the transform $\mathbf{T}_{A \rightarrow B}$ is the affine transformation between coordinate system $A$ and coordinate system $B$.

\subsection{Calibration}

Calibration is a necessary and crucial process that is performed to find the transformation $\mathrm{T}_{D \rightarrow R}$ between the coordinate system attached to the US B-scan plane and the coordinate system of the position sensor. The real-time position of the B-scan plane is used to generate two of the principal displays (see Fig. 2); this position is unknown until we perform the calibration. We have used the single-wall phantom method as described by Prager et al. [7]. The accuracy of the calibration was one of the lower bounds for the final positioning error for the system.

\subsection{Registration}

The registration step is done intraoperatively with the subject placed on the OR table and before the procedure takes place. The registration transformation $\mathbf{T}_{T \rightarrow P}$ is found in two stages: an initial rigid registration and a real time adaptive registration. 
The initial registration is performed by either using anatomical fiducials or high contrast fiducials placed on the skin before the preoperative imaging. Those fiducials are identified in the CT image and in the coordinate system $T$ by touching them with a tracked pointer. The registration matrix is solved with Horn's pair-wise point matching method [8]; the resulting transformation is denoted as $\mathbf{T}^{\text {orig }} \rightarrow P$.

The real time registration is performed by using an additional position sensor, attached to the subject's thorax, as a local reference frame. This real-time adaptive registration compensates for rigid movements of the patient. The registration transformation $\mathbf{T}_{T \rightarrow P}$ is updated according to the expression

$$
\mathbf{T}_{T \rightarrow P}=\mathbf{T}_{T \rightarrow P}^{\text {orig }} \mathbf{T}_{R \rightarrow T}^{\text {init }}\left(\mathbf{T}_{R \rightarrow T}^{m}\right)^{-1}
$$

where $\mathbf{T}_{R \rightarrow T}^{\mathrm{m}}$ is the transformation given by the sensor attached to the patient and $\mathbf{T}^{\text {init }}$ $R \rightarrow T$ is the initial transformation reported by the sensor after it is attached to the patient.

\subsection{Display}

The display consists of three primary elements (see Fig. 2):

- Display 1: A 3D scene of the patient skeleton and principal vascular structures obtained from the preoperative dataset and a model of the tracked endoscopic probe and the position and orientation of the EUS plane.

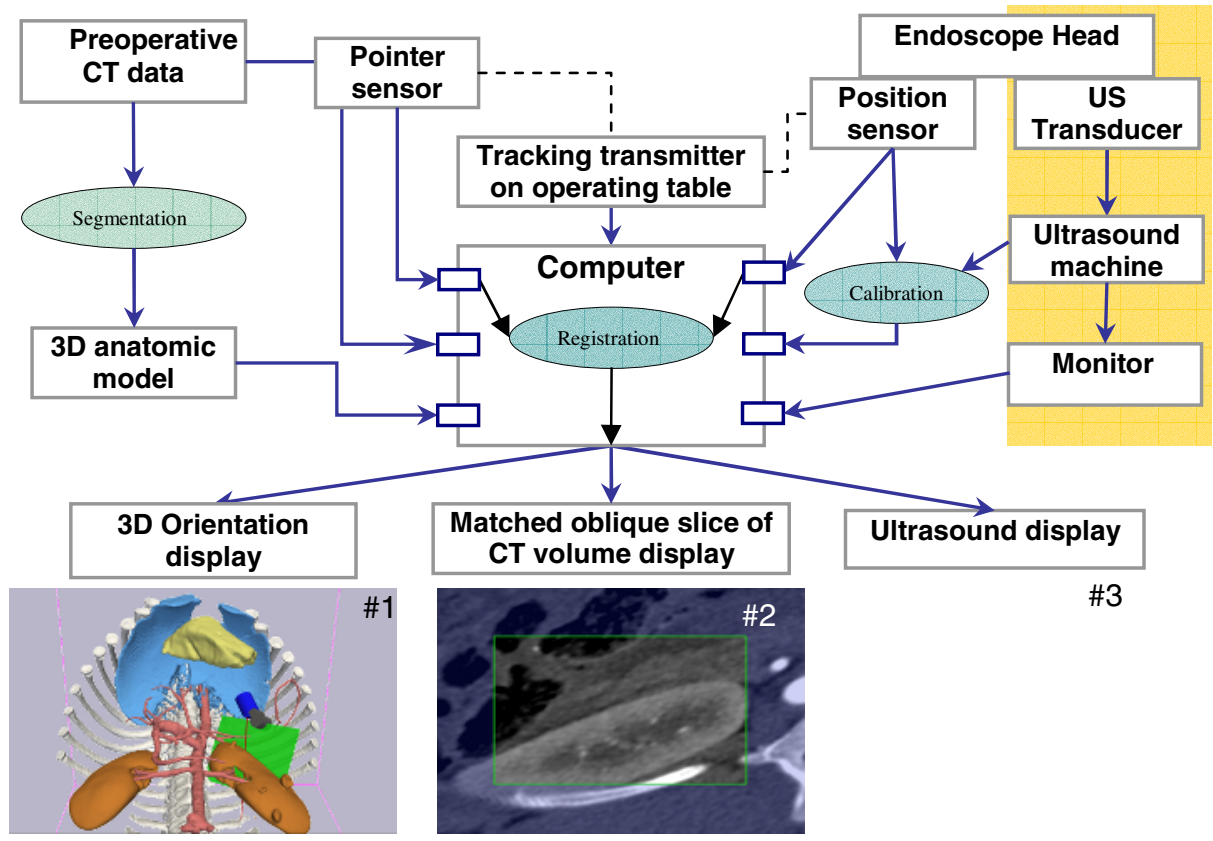

Fig. 2. System description: data flow paths and main displays 
- Display 2: A reformatted CT image in the oblique plane corresponding to the EUS image. The reformat image is augmented by showing a greater extent that the one covered by the US plane. A square outline shows the area corresponding to the US field of view.

- Display 3: Direct acquisition of the EUS image

\section{Materials and Methods}

The tracking system that we have employed is an electromagnetic tracker (MicroBIRD, Ascension Technology Corp., Burlington, VT) which was connected to the computer through a PCI board. The sensor attached to the endoscope tip was $0.3 \mathrm{~mm}$ diameter and $1.8 \mathrm{~mm}$ length. The attachment of the sensor to the ultrasound probe causes an increase in overall probe diameter of less than $2 \mathrm{~mm}$ which is immaterial in the probe use. A similar sensor is used for the real time adaptive rigid registration. The US images were provided by BK Panther Laparoscopic Ultrasound for LUS acquisitions are Olympus EU-C60 for EUS acquisitions. Both ultrasound systems have Doppler capabilities. Preoperative CT were made with a Siemens Sensation 64. Three scans were acquired per study: a baseline scan, a contrast enhanced scan and delayed scan.

Our system was tested in a porcine model under general anesthesia. Free breathing was allowed and forced ventilation was only used during CT scanning to reduce breathing artifacts. Before CT scanning, four high-contrast CT markers were placed on the laterals of the rib cage. The subject was placed on the OR, the fiducial makers were located, and the initial registration was calculated.

To assess the performance of our system, both quantitatively and qualitatively, we ran two set of experiments: one for system error and one for user performance. In all cases we recorded all instrument motions and corresponding US images for retrospective analysis.

\subsection{Experiment 1}

This experiment, using a tracked LUS probe, was designed to assess the total registration error incurred by our system. Laparoscopic or transgastric procedures normally require insufflation of the abdominal cavity. We hypothesize that the distortion due to insufflation would impose a lower bound for the total registration error for these interventions, whether laparoscopic or trans-gastric.

Four landmarks were used as reference to assess the error. Three landmarks were anatomical features, namely, the branching points between the aorta and three major arteries: the celiac, superior mesenteric (SMA) and right renal. The fourth landmark was a $6 \mathrm{~mm}$ diameter bead that was implanted in the left kidney. The bead was clearly observed both in CT and US.

To assess the error, an expert was asked to perform a standard Laparoscopic exploration and to identify several US images where those landmarks were clearly visible. Doppler ultrasound was employed for the vessel landmarks to give an accurate location of the branch point. Independently, the same land-marks were identified in the CT volume. The total registration error was measured as the distance between a CT landmark 


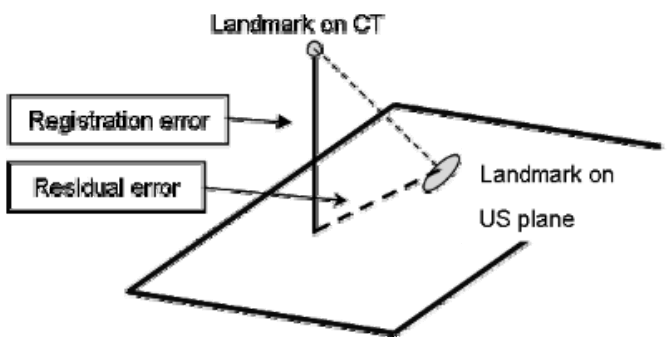

Fig. 3. Registration error definitions

and the US planes where the same landmark was visible (see Fig. 3 4b). The position of the US plane in the CT space was known through the transformations given by our system. We considered the error in the normal direction to the US plane to be primary, and any error within the US plane to be residual. Because our system is intended to provide contextual information, and because the clinician can readily compensate for misalignments between the CT oblique slice and the US plane (as long as the target structure was present in both modalities), the normal error is the limit on how far the clinician needed to search for the target.

We also measured respiratory-induced motion of a target organ by stitching a tracker to the right kidney surface. Tracking data were acquired during free breathing and forced (ventilated) breathing; we recorded the insufflation and the heart rate. Motion data were examined with time-series analysis to find the principal harmonic at that corresponded with the breathing frequency timed during the experiment. After filtering that harmonic in the $\mathrm{X}, \mathrm{Y}$ and $\mathrm{Z}$ time-series, a principal-component analysis (PCA) was performed. The net motion was computed as $2\left(\lambda_{1}\right)^{1 / 2}$ where $\lambda_{1}$ is the principal eigenvalue of the covariance matrix.

\subsection{Experiment 2}

A group of 3 experts and 5 novices in US guided endoscopic interventions were requested to localize a predefined list of targets on a fixed time of 5 minutes. The users ran this task twice, using the conventional EUS technique and using our Image Registered Gastroscopic Ultrasound (IRGUS) system. We interleaved the tasks between users, so half used the IRGUS system first and the other half used the EUS first. During the experiment, the location and orientation of the probe was recorded and we noted which structures were properly identified. Novice users were always assisted by an expert. From the positioning data, a kinematic evaluation of the user's motion was performed to characterize performance during the task [9]. Kinematic analysis provides measurements for the amount and smoothness of motion that is required to achieve the task. Finally, a questionnaire was used at the end of the task to assess subjective responses to the navigation system.

\section{Results}

The total registration error was computed for each landmark using 4 US slices where the landmarks were visible. The average total error is shown in Fig. 4a. The error upper boundary is approximately $5 \mathrm{~mm}$ range. Because the landmark features were also of that size, the landmarks should have always been visible in the reformatted CT plane. We confirmed this by retrospectively evaluating the results: reviewing the data and 
Table 1. Kinematic analysis comparing our system (IRGUS) and conventional endoscopic approach (EUS)

\begin{tabular}{|c|c|c|c|c|}
\cline { 2 - 5 } \multicolumn{1}{c|}{} & Path Length & $\begin{array}{c}\text { Smoothness of } \\
\text { Motion } \\
\mathrm{d}^{3} \mathrm{~d} / \mathrm{dt}^{3}\end{array}$ & $\begin{array}{c}\text { Depth } \\
\text { Perception } \\
(\mathrm{cm})\end{array}$ & $\begin{array}{c}\text { Response } \\
\text { Orientation } \\
\text { (radians) }\end{array}$ \\
\hline EUS & 1600.3 & 12.6 & 9877.5 & 52.5 \\
\hline IRGUS & 1245.7 & 9.2 & 8174.2 & 42.3 \\
\hline
\end{tabular}

(a)

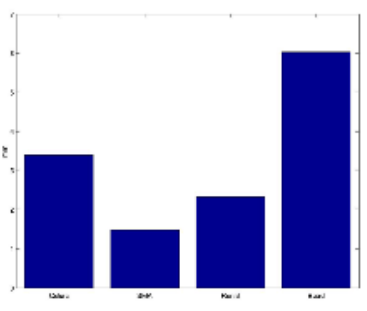

(b)
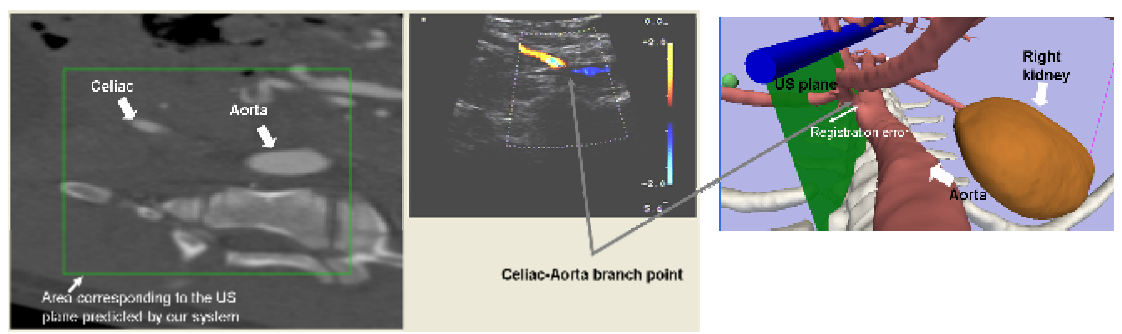

Fig. 4. Registration error for 4 landmarks. (a) Average error for different US planes where the landmarks were visible. (b) Example of one plane used for the evaluation of the error corresponding to the celiac branch.

reconstructing views, we confirmed that all landmarks were visible in both in the US plane and the CT oblique at times when the expert reported localizing the landmarks.

Our data for respiratory-induced kidney motion were acquired during free breathing and ventilation with an insufflation of $200 \mathrm{ml}$. The subject's heart rate was steady at 70 beat/s. The principal harmonic of motion was $0.6 \mathrm{~Hz}$, which corresponds with the breathing frequency that was timed during the experiment. The total displacement in the direction of maximum variance was $1.8 \mathrm{~mm}$ for forced breathing and $1.4 \mathrm{~mm}$ for free breathing.

Our second experiment showed that using the conventional EUS, novices identified only $29 \%$ of the structures and experts $50 \%$ within the allotted time. In contrast, IRGUS significantly increased to $71 \%$ and $80 \%$ respectively. In addition, the analysis of kinematic data showed that using IRGUS the physicians not only identified more structures, but they were more efficient as well (see Table 1). IRGUS improved efficiency of conventional EUS by $17-27 \%$ in the analyzed characteristics. All differences were statistically significant at the level of $\mathrm{p}<0.05$. 


\section{Discussion}

The utility of our system does not depend on absolute spatial precision. Our hypothesis is that the orthogonal distance between the US plane and a target on the preoperative data is on an acceptable range (less than $5 \mathrm{~mm}$ ) to allow context information. We also found that when targets appeared in both the US plane and in the reformatted CT - regardless of the amount of displacement within that plane users found the contextual information very useful in guiding interventions. A major concern was that the motion of organs (such as the kidney) induced by respiration would compromise the utility of our system, but our experiments showed that this motion was limited and was significantly lower than the registration errors

A key component of our approach is that we have relaxed the accuracy requirements for registration of patient's anatomy given by the US to the preoperative volumetric images. This "reference" registration consists on relying on an initial rigid registration of the scanner space to the patient space, plus a real time correction of this initial rigid registration computed by tracking the patient position with a sensor. By complementing real time imaging with closely registered preoperative images, we aim to improve the way in which real-time images are interpreted, but without relying on high accuracy registration methods needed by traditional image-based navigation systems [10]. We claim that reference registration is particularly suited to endoscopic abdominal surgery where, by using preoperative data for context and real-time imaging for targeting, distortions that limit the use of only preoperative data can be overcome. We observed that the accuracy of our approach is within surgically acceptable limits and that the contextual information provided by our navigation system improved the performance of both expert and novice users.

Novice clinicians performing US guided endoscopic interventions found the system easy to master and improved their confidence in the identification of different structures thanks to the closely registration to the CT. The number of structures that they were able to correctly identify was double than without the assistance of the system. Even expert clinicians found the navigation system of great help, increasing their confidence in the imaged structured by the real-time US and reducing their intervention time.

Our system can assist in the implementation of transgastric interventions. One of the major difficulties while performing the intervention is to avoid major stomach vessels when puncturing the stomach wall. This puncture can be performed with minimal bleeding is those vessels are avoided. However, if the vessel is accidentally comprise, fatal iatrogenic injury could lead to the death of the patient. Our system can assist in this crucial task by tracking the position of the tip of the instrument in relation to the vessels of the stomach and other abdominal structures.

\section{Acknowledgments}

This work has been supported by the US Department of the Army under award DAMD 17-02-2-0006 to CIMIT. The information does not necessarily reflect the position of the government and no official endorsement should be inferred. This work has been partially supported by NIH P41-RR13218. The authors want to acknowledge the material and support provided by the following companies: Ascension Technologies, B-K Ultrasound, and Olympus. 


\section{References}

1. M.S. Wagh, B.F. Merrifield, C.C. Thompson, "Survival studies after endoscopic transgastric oophorectomy and tubectomy in a porcine model", Gastrointest. Endosc., vol. 63, pp. 473-478, 2006.

2. J. Harms, H. Feussner, M. Baumgartner, et al, "Three-dimensional navigated laparoscopic ultrasonography”, Surg. Endosc, vol. 15, pp. 1459-1462, 2001.

3. J. Ellsmere, J. Stoll, D. Rattner, D. Brooks, R. Kane, W. Wells, R. Kikinis, and K. Vosburgh, "A Navigation System for Augmenting Laparoscopic Ultrasound", Lectures Notes in Computer Science, vol. 2879, pp. 184-191, 2003.

4. F. Lindseth, S. Ommedal, J. Bang, et al.: "Image Fusion of Ultrasound and MRI as an Aid for assessing Anatomical Shifts and improving overview and interpretation in Ultrasound Guided Neurosurgery". CARS 2001, 1230:247-252, 2001.

5. T. Lange, S. Eulenstein, M. Hunerbein. "Augmenting Intraoperative 3D Ultrsound with Preoperative Models for Navigation in Liver Surgery”. MICCAI 2004 Part II, Saint Malo, Springer LNCS 3216, C Barilot, DR Haynor, Eds, pp. 26-29, 2004.

6. Becker HD, Herth F, Ernst A, Schwarz Y. "Bronchoscopic Biopsy of Peripheral Lung Lesions Under Electromagnetic Guidance: A Pilot Study", J Bronchology, vol. 12, pp. 9-13, 2005.

7. R. Prager, R. Rohling, A. Gee, et al. "Rapid calibration for 3-D freehand ultrasound". Ultrasound Med Biol, vol 24, pp. 855- 869, 1998.

8. B.K.P. Horn, "Closed-form solution of absolute orientation using unit quaternions", Journal of the Optical Society of America A, vol. 4, pp. 629-642. 1987.

9. N. Stylopoulos, S. Cotin, S.K. Maithel, M. Ottensmeyer, P.G. Jackson, R.S. Bardsley, PF Neumann, D.W. Rattner, S.L. Dawson. "Computer-enhanced laparoscopic training system (CELTS): bridging the gap”. Surg Endosc. Vol 18, pp. 782-789, 2004.

10. G. Penney, J. Blackall, M. Hamady, et al. "Registration of freehand 3D ultrasound and magnetic resonance liver images”, Medical Image Analysis, vol. 8, pp. 81-91, 2004. 